\title{
Traditional Chinese Medicine as a Remedy for Male Infertility: A Review
}

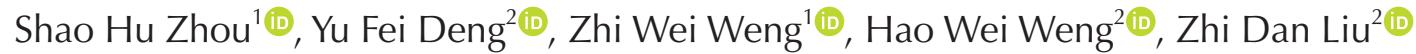 \\ ${ }^{1}$ Department of Reproductive Medicine, The First Affiliated Hospital of Guangzhou University of Chinese Medicine, ${ }^{2}$ The First Clinical \\ Medical College, Guangzhou University of Chinese Medicine, Guangzhou, China
}

\begin{abstract}
Male infertility (MI) is a complex multifactorial disease, and idiopathic infertility accounts for $30 \%$ of cases of MI. At present, the evidence for the effectiveness of empirical drugs is limited, and in vitro fertilization is costly and may increase the risk of birth defects and childhood cancers. Therefore, affected individuals may feel obliged to pursue natural remedies. Traditional Chinese medicine (TCM) may represent a useful option for infertile men. It has been demonstrated that TCM can regulate the hypothalamic-pituitary-testicular axis and boost the function of Sertoli cells and Leydig cells. TCM can also alleviate inflammation, prevent oxidative stress, reduce the DNA fragmentation index, and modulate the proliferation and apoptosis of germ cells. Furthermore, TCM can supply trace elements and vitamins, ameliorate the microcirculation of the testis, decrease the levels of serum anti-sperm antibody, and modify epigenetic markers. However, the evidence in favor of TCM is not compelling, which has hindered the development of TCM. This review attempts to elucidate the underlying therapeutic mechanisms of TCM. We also explore the advantages of TCM, differences between TCM and Western medicine, and problems in existing studies. Subsequently, we propose solutions to these problems and present perspectives for the future development of TCM.
\end{abstract}

Keywords: Infertility, male; Medicine, Chinese traditional; Therapeutics

This is an Open Access article distributed under the terms of the Creative Commons Attribution Non-Commercial License (http://creativecommons.org/licenses/by-nc/4.0) which permits unrestricted non-commercial use, distribution, and reproduction in any medium, provided the original work is properly cited.

\section{INTRODUCTION}

Infertility is defined as no conception after at least of 12 months of unprotected intercourse. Infertility affects up to $15 \%$ of couples, and male fertility has been found to be deficient in no fewer than $50 \%$ of infertile couples [1]. According to the 2012 publication of the European Association of Urology Guidelines on Male Infertility (MI), 30\% of infertile men suffer from idiopathic infertility [2]. However, evidence-based data are limited in the empirical use of drugs for idiopathic MI
[3], and some drugs have obvious side effects.

The treatment of MI has been revolutionized by advances in assisted reproductive technology, including intrauterine insemination, in vitro fertilization (IVF) and even intracytoplasmic sperm injection (ICSI). However, IVF and ICSI procedures are costly and traumatic. Additionally, the use of IVF and/or ICSI techniques may increase the risk of birth defects and childhood cancers in the offspring. Therefore, seeking effective natural remedies to enhance fertility is still the principal alternative for most people affected by infertil-

Received: Aug 1, 2018 Revised: Oct 13, 2018 Accepted: Oct 17, 2018 Published online Jan 10, 2019

Correspondence to: Shao Hu Zhou (iD https://orcid.org/0000-0002-1939-2783

Department of Reproductive Medicine, The First Affiliated Hospital of Guangzhou University of Chinese Medicine, 16 Airport Road, Baiyun District, Guangzhou, 510405 Guangdong, China.

Tel: +86-020-36591572, Fax: +86-020-88773879, E-mail: zhoushaohu@vip.163.com 
ity. In China, Traditional Chinese medicine (TCM) has been employed to treat MI for more than 2000 years, which has influenced the opinions of people in surrounding areas and has made it increasingly appealing to people with infertility. Holism and treatments based on syndrome differentiation are the essence and the basic characteristics of TCM, and utilizing this theory for treating MI yields satisfactory results.

However, the theory of TCM is abstract, and the therapeutic mechanism of TCM is obscure, which has impeded its popularization and aroused skepticism among Western scientists regarding its potency. This review sheds light on the potential therapeutic mechanisms of TCM and includes information regarding the advantages of TCM, the differences between TCM and Western medicine, and problems with existing studies. Subsequently, we propose solutions to the problems and present perspectives for the future development of TCM.

\section{POTENTIAL THERAPEUTIC MECHANISMS OF TRADITIONAL CHINESE MEDICINE}

\section{Regulation of the reproductive endocrine system}

In the theory of TCM, the kidney stores an individual's essence and governs growth, development, and reproduction. TCM regards the kidney as the prenatal source of life; therefore, the fundamental pathogenesis of MI is kidney deficiency, and supplementing the kidney is recognized as the essential therapy for MI. The hypothalamic-pituitary-testicular (H-P-T) axis is a major positive- and negative-endocrine feedback system that regulates testis function. Hormone levels that are either too high or too low are detrimental to spermatogenesis. Modern research has demonstrated that kidney deficiency often manifests with the dysfunction and impaired structure of the H-P-T axis [4]. Experiments have shown that kidney-supplementing formulas could repair the structure and restore the function of the H-P-T axis [5], bidirectionally regulate the hormone levels of follicle-stimulating hormone (FSH) and luteinizing hormone (LH), and eventually increase the level of testosterone ( $\mathrm{T}$ ) to improve the quality of semen.

\section{1) Enhancement of follicle-stimulating hormone} levels, regulation of luteinizing hormone levels

Recent studies have indicated that a Chinese herbal monomer regulates the levels of FSH and LH. Lycium barbarum, a traditional Chinese medicinal herb, has been used to enhance male fertility for thousands of years, and L. barbarum polysaccharide (LBP) is the major active component isolated from L. barbarum. Animal experiments have shown that LBP could significantly raise FSH and LH levels in heat-induced model rats [6]. Ginsenosides and their monomers of Rb1 and Rg1 were able to stimulate cultured anterior pituitary cells to secrete FSH and LH in vitro [7]. Additionally, semen Cuscutae extracts significantly improved FSH and LH levels in adenine-induced model rats [8]. Chinese herbal compounds also have had the same effect. Studies have found that the liuwei dihuang pill significantly increased FSH and LH levels in kidney-yindeficient infertile men [9]. Furthermore, the wuzi yanzong pill (WYP) was found to promote the secretion of FSH and to simultaneously reduce LH levels through a negative feedback pathway [10]. Low levels of FSH imply hypospermatogenesis, and kidney-supplementing and replenishing herbal medicines heightened testicular function in spermatogenesis through upregulation of FSH.

2) Reducing follicle-stimulating hormone levels, regulating luteinizing hormone levels

Previous data suggest that Schizandra chinensis polysaccharide extracted from $S$. fructus reduced FSH and LH levels in cyclophosphamide-induced model rats [11]. In addition, animal experiments confirmed that the jingui shenqi pill remarkably lowered FSH and LH levels in adenine-induced kidney-yang-deficiency rats [12]. Moreover, the liuwei dihuang decoction was capable of decreasing FSH levels and increasing LH levels in model rats treated with gossypol acetate [13]. Abnormally high FSH and LH levels suggest injured spermatogenesis in the testis, and kidney-supplementing and essence-replenishing herbal medicines can repair the damaged histologic structure of the seminiferous epithelium and stimulate spermatogenesis by downregulating FSH levels.

\section{3) Raising testosterone levels}

In addition to regulating the H-P-T axis to raise $\mathrm{T}$ levels [8-13], modern pharmacological studies have 
shown that echinacoside extracted from Cistanche tubulosa and Cistanche tubulosa itself significantly enhanced testosterone biosynthesis by increasing the expression of key steroidogenic enzymes, including steroidogenic acute regulatory protein (StAR), cytochrome P450 side chain cleavage (CYP11A1), 33-hydroxysteroid dehydrogenase (HSD), 17ß-HSD, and CYP17A1 [14]. This suggests that kidney-supplementing herbal medicines elevate $\mathrm{T}$ levels via multiple targets and pathways.

\section{4) Regulating follicle-stimulating hormone and} luteinizing hormone levels bidirectionally

A prospective controlled study showed that the bushen shengjing pill increased LH levels in kidneyyang-deficient infertile men, and decreased FSH levels in kidney-yin-deficient and kidney-yin and -yangdeficient men [15]. The results from this study and the studies mentioned in the previous sections suggest that TCM regulates FSH and LH level bidirectionally, maintaining endocrine homeostasis.

\section{Boosting the function of Sertoli cells and Leydig cells}

Sertoli cells (SCs) play a central role in spermatogenesis. A reduction in the number of $\mathrm{SCs}$ leads to a proportional decrease in the number of germ cells and Leydig cells (LCs), with detrimental effects on fertility [16]. SCs secrete androgen-binding protein under the regulation of FSH, while LH stimulates LCs to synthesize T. These 2 processes cooperate to maintain normal spermatogenesis. Experiments have shown that TCM promoted the proliferation of SCs and LCs, elevated the levels of inhibin B and T, and eventually facilitated the progression of germ cells to spermatozoa.

The WYP is an essential formula for the treatment of MI. Previous studies have demonstrated that the WYP could dramatically enhance the activity of cytochrome c oxidase in SCs [17] and inhibit the overexpression of Cox7a2 in SCs [18]. Additionally, the WYP prominently elevated levels of serum inhibin B in model rats induced by multiglycosides of Tripterygium wilfordii Hook F [19]. WYP treatment also increased vimentin expression and repaired the cytoskeleton in SCs [20], and additionally activated the Akt signal transduction pathway and upregulated p-Akt expression to induce proliferation of SCs [21]. Notably, liuwei dihuang pill extract was also capable of stimulating SCs to proliferate [22].
Previous studies have suggested that yangjing capsule (YC) extract could significantly upregulate testosterone synthesis in LCs via the Nur77 pathway [23]. Moreover, icariin treatment significantly reduced apoptosis and promoted the proliferation of LCs [24]. Meanwhile, icariin was found to stimulate the proliferation of SCs by activating the ERK1/2 signal pathway and upregulating the mRNA expression of FSH receptor and claudin-11 in SCs [25,26].

\section{Preventing oxidative stress}

In recent decades, problems including infection, environment pollution, and lifestyle have increased, and cellular damage caused by oxidative stress (OS) has attracted attention. Excessive reactive oxygen species (ROS) attack the membrane and DNA of sperm cells, leading to decreased fluidity and impeded permeability of the sperm plasma membrane. This results in an augmented DNA fragmentation index (DFI) and apoptosis rate, ultimately resulting in infertility. TCM has been demonstrated to scavenge ROS, improve the antioxidant capacity of the seminal plasma, lower sperm DFI, and protect the male reproductive system from the lesions induced by ROS.

Pharmacological studies have indicated that Morinda officinalis, semen Cuscutae, Lycii fructus, and Schisandrae fructus could increase superoxide dismutase (SOD) levels and reduce the malondialdehyde (MDA) content in the testis to protect the sperm from OS [27-30]. Wuzi yanzong compound extract improved the survival rate of SCs, elevated the activity of SOD, and reduced the MDA content in SCs injured by $\mathrm{H}_{2} \mathrm{O}_{2}$ [31]. The jinkui shenqi pill increased the activity of SOD and decreased MDA levels in serum from model rats with infertility induced by hydrocortisone [32]. Furthermore, experiments by Zhou et al [33] and Weng et al [34] on rats induced by cadmium chloride and cigarette smoke indicated that kidney-supplementing and blood-quickening formulas were capable of significantly raising the activity of SOD and glutathione reductase (GSH) in the testis, while lowering MDA levels and sperm DFI [33,34].

OS is a common consequence of spermatogenic damage, and TCM remedies MI by means of antioxidation.

\section{Modulating the proliferation and apoptosis of germ cells}

SCs are the paracrine regulators of spermatogenesis 
and secrete glial cell line-derived neurotrophic factor (GDNF), which is an important growth factor that modulates the differentiation and self-renewal of spermatogonia [35]. Animal experiments have shown that Cynomorium songaricum extracts dramatically increased the expression of GDNF (mRNA and protein) in SCs [36]. In vitro cell culture experiments indicated that YC extract could act on GDNF to upregulate POU3F1 expression by triggering the activation of the PI3K/AKT pathway, ultimately stimulating spermatogonial stem cell proliferation [37]. Furthermore, matrimony vine treatment was able to facilitate the division and differentiation of spermatogonia and propagation of primary spermatocytes [38].

Apoptosis is commonly known as programmed cell death. The apoptosis of germ cells is an important testicular self-regulation process. However, high levels of apoptosis cause MI. Researchers have suggested that LBP decreased the expression levels of caspase-3, increased the Bcl-2/Bax-43 ratio and the testicular antioxidant enzyme activity of SOD and GSH, and eventually inhibited the apoptosis of testicular germ cells in streptozotocin-induced diabetic mice [29]. The jingui shenqi pill reduced the expression of Fas/FasL to inhibit germ cell apoptosis in a kidney-yang-deficiency mice model [39]. Furthermore, wuzi yanzong formula treatment downregulated protein expression levels of Bax and caspase-3 [40], and upregulated the expression of Bcl-2, which modulates the p53 signal pathway and the H-P-T axis. Ultimately, T levels were increased, inhibiting apoptosis in germ cells [41,42].

Therefore, TCM prompts the proliferation of spermatogonia and inhibits the apoptosis of germ cells to treat MI.

\section{Supplementing trace elements}

Zinc and selenium are essential trace elements for testicular development and spermatogenesis. Zinc is a component of SOD and plays an important role in antioxidation [43], DNA repair mechanisms, and maintaining genomic stability [44]. Selenium is a critical component of phospholipid hydroperoxide glutathione peroxidase, which shields membrane lipids from oxidation. Selenium is also a constituent of the mitochondrial sheath of spermatozoa [45]. Manganese is also an essential trace element that acts as a potent trigger of sperm motility by increasing adenylate cyclase activity [46]. Substantial evidence has demonstrated that infertile men are likely to have a zinc or selenium deficiency, and moderate supplementation of zinc and selenium improved the semen quality of infertile men $[47,48]$. Domestic studies have shown that kidneysupplementing herbal medicines such as Epimedium and Curculigo were rich in zinc and manganese [49], while cooked Rehmannia had high levels of selenium [50]. Therefore, TCM supplements zinc, selenium, and manganese to remedy $\mathrm{MI}$.

\section{Ameliorating the microcirculation of the testis}

Spermatogenesis is a highly metabolic process that is susceptible to disruptions in the supply of nutrients and oxygen. Sufficient blood supply for the testis is a prerequisite for normal spermatogenesis. Previous studies have advanced the concept of treating male disease from the perspective of blood stasis [51]. Zhou and Xie [51] insisted that blood-quickening medicines ameliorated the microcirculation and metabolism of the testis and ensured an adequate nutrient supply for the testis. Blood-quickening medicine alleviated inflammatory effusion and inflammation and unblocked the vas deferens.

Animal experiments have suggested that bushen huoxue formulary treatment could upregulate the protein expression of vascular endothelial growth factor (VEGF) receptor 2 (VEGFR2) and Rous sarcoma oncogene (Src) via the VEGF/VEGFR2 pathway to facilitate testicular microcirculation in cyclophosphamide model mice [52]. A randomized controlled trial of 128 infertile men indicated that kidney-supplementing and blood-quickening methods dramatically raised sperm concentration and motility more effectively than other therapeutic methods [53]. Kidney-supplementing and blood-quickening formulas also improved semen quality and pregnancy rate.

\section{Improving semen quality and the pregnancy rate}

\section{1) Improving seminal plasma quality}

Fructose and alpha-glucosidase are the major components of seminal plasma. Fructose is the energy source for sperm; it is metabolized into adenosine triphosphate by glycolysis and is associated with sperm motility. Alpha-glucosidase can catalyze the degradation of the glycogen stores of sperm in the epididymis, supplying 
energy for the maturation and motion of sperm. Studies by Chen et al [54] found that jiawei wuziyanzong decoction treatment increased fructose levels in seminal plasma in infertile men with asthenozoospermia. We thought that seed herbal medicines contained numerous vitamins and fructose and could elevate the fructose level in seminal plasma, consequently improving sperm motility. Another study showed that shengjing prescription treatment significantly increased the levels of seminal plasma alpha-glucosidase and fructose [55]. Therefore, TCM can elevate sperm motility by increasing fructose and alpha-glucosidase levels in seminal plasma.

\section{2) Improving sperm quality}

A randomized controlled study showed that both jiawei wuzi yanzong decoction and liuwei dihuang pill treatments improved sperm vitality and motility in kidney-yin-deficient infertile men [9], which is consistent with previous studies. A multicenter study found that both the qilin pill and the WYP could effectively improve the seminal concentration and sperm motility in oligoasthenozoospermia patients, which is in accordance with the results from treatment with the shengjing prescription [56]. In addition, shengjing prescription treatment improved normal sperm morphology and acrosin activity, reducing sperm DFI [55]. Because of the differences in basic features of the included studies, 2 meta-analyses yielded inconsistent results. One metaanalysis suggested that TCM could improve sperm concentration, sperm motility, and the pregnancy rate more effectively than Western medicine [57]. However, another meta-analysis indicated that TCM and ChineseWestern combined therapy significantly enhanced the pregnancy rate. That study only performed a descriptive analysis on semen parameters due to the high heterogeneity of the included studies [58]. In terms of the efficacy of acupuncture, a meta-analysis showed that acupuncture was able to increase grade A sperm motility and sperm concentration, but had no significant impact on the pregnancy rate [59].

\section{Others}

\section{1) Alleviating inflammation}

Mycoplasma and Chlamydia infections of the male genital tract are a common etiology of $\mathrm{MI}$ [60], resulting in decreased sperm motility and increased abnor- mal sperm counts. Inflammation leads to leukocyte infiltration into the seminal plasma, and the leukocytes release excessive ROS, which attack the plasma membrane and DNA of sperm. Experiments have shown that Phellodendron, Scutellaria, and Taraxacum were effective at killing mycoplasma and chlamydia in vitro, while zhibai dihuang decoction treatment increased protein and mRNA expression levels of interleukin-2 and inhibited the protein and mRNA expression of tumor necrosis factor alpha in rats infected by Ureaplasma urealyticum [61]. Hence, TCM not only kills pathogenic microorganisms directly, but also regulates cytokine secretion to treat infections indirectly.

\section{2) Decreasing the level of anti-sperm antibody}

Sperm is a specific antigen that causes the human body to generate anti-sperm antibody (AsAb) when the immune system is exposed to it. AsAb decreases sperm motility [62], impedes sperm from undergoing capacitation and acrosome reactions, and interferes with sperm-oocyte recognition and fusion [63]. Animal experiments have suggested that zhibai dihuang decoction treatment could remarkably reduce serum levels of AsAb in rats [64]. While the concrete mechanism is obscure, TCM may eliminate testicular immunological complexes and regulate the ratio of $\mathrm{CD}_{4} / \mathrm{CD}_{8} \mathrm{~T}$ cells to cure immune-induced infertility $[65,66]$.

\section{3) Modifying epigenetic markers}

Epigenetics is the study of modification of gene expression without changing the DNA sequence, and epigenetic processes include DNA methylation, histone modification, and chromatin remodeling [67]. Normal H19 gene expression is crucial to spermatogenesis, and many patients with oligoasthenoteratozoospermia had hypomethylation at the H19 locus [68]. A study by Lian et al [69] indicated that shengjing formula treatment could improve sperm concentration, motility and clinical pregnancy rate by reducing the loss rate of the H19 imprinted gene. Research into TCM in the field of epigenetics is limited and needs to be strengthened.

\section{CONCLUSIONS}

Overall, the effectiveness of TCM for MI has been confirmed by numerous studies, but many problems exist in these studies. The advantages of TCM and the differences between TCM and Western medicine, as 
Table 1. Potential therapeutic mechanisms of TCM

\begin{tabular}{|c|c|c|}
\hline Function of TCM & Chinese herbal medicines and monomers & Potential therapeutic mechanisms of TCM \\
\hline \multicolumn{3}{|c|}{ Regulating the reproductive endocrine system } \\
\hline \multirow[t]{4}{*}{$\begin{array}{l}\text { Enhancing FSH levels, } \\
\text { regulating LH levels }\end{array}$} & Ginsenosides and Rb1, Rg1 & $\begin{array}{l}\text { Stimulated cultured anterior pituitary cells to secrete FSH and } \\
\text { LH in vitro }\end{array}$ \\
\hline & LBP, semen Cuscutae extract & \\
\hline & Liuwei dihuang pill & Significantly increased FSH and LH levels \\
\hline & WYP & $\begin{array}{l}\text { Promoted the secretion of FSH and simultaneously reduced LH } \\
\text { levels }\end{array}$ \\
\hline \multirow{3}{*}{$\begin{array}{l}\text { Reducing FSH levels, regulating } \\
\text { LH levels }\end{array}$} & Schizandra chinensis polysaccharide & \\
\hline & Jingui shenqi pill & Reduced FSH and LH levels \\
\hline & Liuwei dihuang decoction & Decreased FSH levels and increased LH levels \\
\hline Raising T levels & Cistanche tubulosa & $\begin{array}{l}\text { Enhanced testosterone biosynthesis by increasing the } \\
\text { expression of key steroidogenic enzymes, including StAR, } \\
\text { CYP11A1, 3 } \beta-H S D, 17 \beta-H S D \text {, and CYP17A1 }\end{array}$ \\
\hline $\begin{array}{l}\text { Regulating FSH and LH levels } \\
\text { bidirectionally }\end{array}$ & Bushen shengjing pill & $\begin{array}{l}\text { Increased LH levels in kidney-yang-deficient infertile men, and } \\
\text { decreased FSH levels in kidney-yin-deficient and kidney-yin } \\
\text { and -yang-deficient men }\end{array}$ \\
\hline \multirow[t]{4}{*}{$\begin{array}{l}\text { Boosting the function of SCs } \\
\text { and LCs }\end{array}$} & WYP & $\begin{array}{l}\text { Enhanced the activity of cytochrome c oxidase, inhibited the } \\
\text { overexpression of Cox7a2 in SCs, repaired the cytoskeleton of } \\
\text { SCs, upregulated p-Akt expression }\end{array}$ \\
\hline & Liuwei dihuang pill extract & Stimulated SCs to proliferate \\
\hline & YC extract & $\begin{array}{l}\text { Upregulated testosterone synthesis in LCs via the Nur77 } \\
\text { pathway }\end{array}$ \\
\hline & Icariin & $\begin{array}{l}\text { Reduced apoptosis and promoted the proliferation of LCs, } \\
\text { stimulated the proliferation of SCs by activating the ERK } 1 / 2 \\
\text { signal pathway and upregulating the mRNA expression of } \\
\text { FSH receptor and claudin- } 11 \text { in SCs }\end{array}$ \\
\hline \multirow[t]{5}{*}{ Preventing oxidative stress } & Morinda officinalis, Cuscutae semen & \\
\hline & Lycii fructus, Schisandrae fructus & Increased SOD levels, reduced the MDA content in the testis \\
\hline & Wuzi yanzong & $\begin{array}{l}\text { Elevated the activity of SOD, and reduced the MDA content in } \\
\text { SCs }\end{array}$ \\
\hline & Jinkui shenqi pill & $\begin{array}{l}\text { Increased the activity of SOD and decreased MDA levels in } \\
\text { serum }\end{array}$ \\
\hline & Qilin pill & $\begin{array}{l}\text { Raised the activity of SOD and GSH in the testis, lowered the } \\
\text { MDA level and the sperm DFI }\end{array}$ \\
\hline \multicolumn{3}{|c|}{ Modulating the proliferation and apoptosis of germ cells } \\
\hline \multirow{3}{*}{$\begin{array}{l}\text { Promptig the proliferation of } \\
\text { spermatogonia }\end{array}$} & Cynomorium songaricum extracts & Increased the expression of GDNF (mRNA and protein) in SCs \\
\hline & Matrimony vine & $\begin{array}{l}\text { Facilitated the division and differentiation of spermatogonia } \\
\text { and propagation of primary spermatocyte }\end{array}$ \\
\hline & YC extract & $\begin{array}{l}\text { Activated the PI3K/AKT pathway, upregulated POU3F1 } \\
\text { expression, ultimately stimulated the spermatogonial stem } \\
\text { cells proliferation }\end{array}$ \\
\hline \multirow[t]{3}{*}{$\begin{array}{l}\text { Inhibiting the apoptosis of } \\
\text { germ cells }\end{array}$} & LBP & $\begin{array}{l}\text { Decreased the expression of caspase- } 3 \text {, increased the } \mathrm{Bcl}-2 \text { / } \\
\text { Bax } 43 \text { ratio, and the testicular enzyme activity of SOD, GSH }\end{array}$ \\
\hline & Jingui shenqi pill & Reduced the expression of Fas/FasL \\
\hline & Wuzi yanzong formula & $\begin{array}{l}\text { Downregulated the protein expression of Bax and Caspase-3, } \\
\text { upregulated the expression of } \mathrm{Bcl}-2 \text {, modulated p53 signal } \\
\text { pathway, enhanced T levels }\end{array}$ \\
\hline \multirow[t]{2}{*}{ Supplementing trace elements } & Epimedium, Curculigo & Were rich in zinc and manganese \\
\hline & Cooked Rehmannia & Had high levels of selenium \\
\hline
\end{tabular}


Table 1. Continued

\begin{tabular}{|c|c|c|}
\hline Function of TCM & Chinese herbal medicines and monomers & Potential therapeutic mechanisms of TCM \\
\hline \multirow[t]{2}{*}{$\begin{array}{l}\text { Ameliorating the } \\
\text { microcirculation of the testis }\end{array}$} & Bushen huoxue formular & $\begin{array}{l}\text { Upregulated the protein expression of VEGFR2 and Src via the } \\
\text { VEGF/VEGFR2 pathway }\end{array}$ \\
\hline & Shengjing decoction II & $\begin{array}{l}\text { Ameliorated the microcirculation of the testis and ensured an } \\
\text { adequate nutrient supply for the testis }\end{array}$ \\
\hline \multicolumn{3}{|c|}{ Improving the semen quality and pregnancy rate } \\
\hline \multirow{2}{*}{$\begin{array}{l}\text { Improving seminal plasma } \\
\text { quality }\end{array}$} & Jiawei wuziyanzong decoction & \\
\hline & Shengjing prescription & $\begin{array}{l}\text { Increased fructose and alpha-glucosidase levels in seminal } \\
\text { plasma }\end{array}$ \\
\hline Improving sperm quality & Qilin pill, WYP, Shengjing prescription & $\begin{array}{l}\text { Improved sperm concentration, motility, normal sperm } \\
\text { morphology, acrosin activity, and reduced sperm DFI }\end{array}$ \\
\hline \multicolumn{3}{|l|}{ Others } \\
\hline \multirow[t]{2}{*}{ Alleviating the inflammation } & Phellodendron, Scutellaria, Taraxacum & Were effective at killing Mycoplasma and Chlamydia in vitro \\
\hline & Zhibai dihuang decoction & $\begin{array}{l}\text { Increased the protein and mRNA expression of IL-2, inhibited } \\
\text { the protein and mRNA expression of TNF- } \alpha\end{array}$ \\
\hline \multirow{3}{*}{$\begin{array}{l}\text { Decreasing the level of } \\
\text { anti-sperm antibody }\end{array}$} & Zhibai dihuang decoction & Remarkably reduced serum level of AsAb \\
\hline & Mianbu formula I & Eliminated testicular immunological complex \\
\hline & Acupuncture & Regulated the ratio of $\mathrm{CD}_{4} / \mathrm{CD}_{8} \mathrm{~T}$ cell \\
\hline Modify epigenetic markers & Shengjing formula & Reduced the loss rate of $\mathrm{H} 19$ imprinted gene \\
\hline
\end{tabular}

TCM: Traditional Chinese medicine, FSH: follicle-stimulating hormone, LH: luteinizing hormone, T: testosterone, LBP: Lycium barbarum polysaccharide, WYP: wuzi yanzong pill, StAR: steroidogenic acute regulatory protein, CYP11A1: cytochrome P450 side chain cleavage, HSD: hydroxysteroid dehydrogenase, SCs: Sertoli cells, LCs: Leydig cells, YC: yangjing capsule, SOD: superoxide dismutase, MDA: malondialdehyde, GSH: glutathione reductase, DFI: DNA fragmentation index, GDNF: glial cell line-derived neurotrophic factor, VEGFR2: vascular endothelial growth factor receptor 2, Src: Rous sarcoma oncogene, VEGF: vascular endothelial growth factor, IL: interleukin, TNF: tumor necrosis factor, AsAb: anti-sperm antibody.

well as unsolved problems and solutions, are summarized below.

\section{Advantages of Traditional Chinese medicine and potential mechanisms}

We propose the following potential mechanisms of TCM treatments of MI: regulation of the H-P-T axis, boosts to the function of SCs and LCs, alleviation of inflammation, prevention of OS, and reduction of the DFI. TCM also acts to modulate the proliferation and apoptosis of germ cells, supply trace elements and vitamins, ameliorate the microcirculation of the testis, decrease AsAb levels, and modify epigenetic markers (Table 1, Fig. 1). TCM has effects on multiple targets, systems, and pathways to improve sperm parameters and the pregnancy rate. The therapy of TCM focuses on overall balance by improving the condition of the body and regulating the testis, instead of complementing a certain hormone directly. Moreover, natural products have few side effects. This may be the underlying mechanism for the bidirectional effects of TCM on disordered hormone levels.

\section{Problems and insufficiency}

At present, however, the evidence in favor of TCM is not compelling. The studies of TCM for MI are almost all domestic and contain small sample sizes. They are also not blinded randomized controlled trials, and do not contain detailed descriptions of the methods of random sequence generation and allocation concealment; therefore, the overall quality score for methodology is low. The absence of uniform standards for the evaluation of TCM efficacy causes considerable heterogeneity. In addition, most TCM studies lack reports of adverse effects and mainly target infertile men with oligoasthenozoospermia, neglecting patients with azoospermia and teratozoospermia. Additionally, the extraction of effective components and reforms in the preparation of herbal medicines are lagging, which hinder its development and popularization.

\section{Solutions and outlook}

In our view, evidence-based-medicine methods must be applied to assess the efficacy of TCM in modern times. The refinement of herbal medicine needs to be promoted. Large-scale, multicenter, and nationalized 


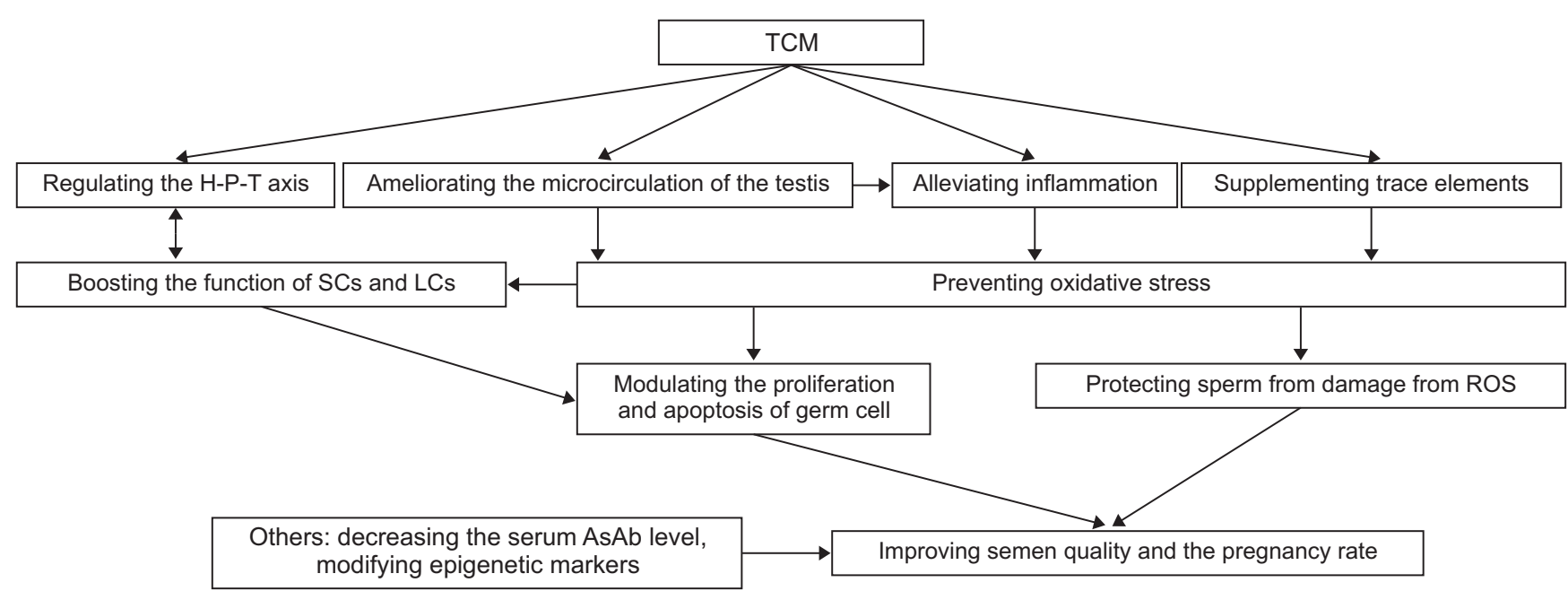

Fig. 1. Potential therapeutic mechanisms of TCM. TCM: Traditional Chinese medicine, H-P-T: hypothalamic-pituitary-testicular, SCs: Sertoli cells, LCs: Leydig cells, ROS: reactive oxygen species, AsAb: anti-sperm antibody.

rigorous randomized controlled trials and further investigations of Chinese herbal monomers will elucidate their therapeutic mechanisms at the molecular and cellular levels, verifying the effectiveness of TCM.

\section{ACKNOWLEDGEMENTS}

The authors thank Weiping Liu, a graduate student of Guangzhou University of Chinese Medicine, for her assistance in this study.

\section{Disclosure}

The authors have no potential conflicts of interest to disclose.

\section{Author Contribution}

Conceptualization, Design and Methodology: Zhou SH. Writing-original draft and Editing: Deng YF. Writing-review: Deng YF. Critical revision of the manuscript: all authors. Approval of final manuscript: all authors.

\section{REFERENCES}

1. Rowe PJ. WHO manual for the standardized investigation, diagnosis and management of the infertile male. 5th ed. Cambridge: Cambridge University Press; 2000;1-5.

2. Jungwirth A, Giwercman A, Tournaye H, Diemer T, Kopa Z, Dohle G, et al. European Association of Urology guidelines on male infertility: the 2012 update. Eur Urol 2012;62:324-32.

3. Garg H, Kumar R. Empirical drug therapy for idiopathic male infertility: what is the new evidence? Urology 2015;86:106575.

4. Cen YH, Zhao FL, Fan R, He GZ. Research progress in the reproductive-related kidney deficiency and kidney tonifying Chinese medicine mechanism. Med Recapitulate 2014;20:2226-8.

5. Qian FL, Zeng FH, Feng WQ. Effects of Chinese herbal preparation for invigorating kidney on function of hypothalamicpituitary-gonad axis in rats. Chin J Sports Med 2005;24:5715.

6. Luo Q, Li Z, Huang X, Yan J, Zhang S, Cai YZ. Lycium barbarum polysaccharides: protective effects against heatinduced damage of rat testes and $\mathrm{H} 2 \mathrm{O} 2$-induced DNA damage in mouse testicular cells and beneficial effect on sexual behavior and reproductive function of hemicastrated rats. Life Sci 2006;79:613-21.

7. Li X, Liu S, Ma X, Xu J. A study of the effect of ginsenosides on the secretion of gonadotropins. J Norman Bethune Univ Med Sci 1988;14:293-5.

8. Nan YY, Wang ZR, Lu ZF, Liu ML, Ma L, Li R, et al. Expression of P450arom CYP19 gonadal hormone levels and influence of the number of spermium in the testis of infertile rats with kidney-yang deficiency after treated by extractive of Cuscuta. J Liaoning Univ Tradit Chin Med 2012;14:20-5.

9. Ye Z, Chen D, Zhong J, Zhang Y, Zhang W, Wang G, et al. Effect of Jiawei Wuzi Yanzong decoction on sperm quality and hormone level. World Chin Med 2013;8:626-9.

10. Xie J, Wang J, Chen M, Liu H. Influence of Guilingji capsules on spermatogenesis and sexual hormones in oligospermatism rats. J Guangzhou Univ Tradit Chin Med 2011;28:621-3.

11. Zhang Y, Shen N, Qi L, Chen W, Dong Z, Zhao DH. Efficacy 
of Schizandra chinesis polysaccharide on cyclophosphamide induced dyszoospermia of rats and its effects on reproductive hormones. Chin J Integr Tradit West Med 2013;33:361-4.

12. Ma L, Jia M, Nan YY, Liu ML, Wang ZR, Ma J. Effects of Jingui Shenqi pills on sperm quality and contents of hormones in adenine-induced infertility rats. J Shandong Univ Tradit Chin Med 2011;35:431-3.

13. Ling Q, Ao Z, Xu Z, Xu Z, Yin G, Tao W. Effect of Liuweidihuang decoction on reproductive system of male rat. Chin Tradit Pat Med 2004;26:561-4.

14. Jiang Z, Wang J, Li X, Zhang X. Echinacoside and Cistanche tubulosa (Schenk) R. wight ameliorate bisphenol A-induced testicular and sperm damage in rats through gonad axis regulated steroidogenic enzymes. J Ethnopharmacol 2016;193:3218.

15. Yue GP, Chen Q, Dai N. Eighty-seven cases of male infertility treated by bushen shengjing pill in clinical observation and evaluation on its curative effect. Chin J Integr Tradit West Med 1996;16:463-6.

16. Rebourcet D, Darbey A, Monteiro A, Soffientini U, Tsai YT, Handel I, et al. Sertoli cell number defines and predicts germ and leydig cell population sizes in the adult mouse testis. Endocrinology 2017;158:2955-69.

17. Yang A, Liu B, Zhang S, Xie C, Li L, Zhou Q, et al. Mechanism of Wuziyanzong Pills in improvement of function of Sertoli cells in rats with insufficiency of kidney essence. J Beijing Univ Tradit Chin Med 2010;33:378-80, 384.

18. Zhang SQ, Liu BX, Wang X, Li LQ, Zhang XP, Zhang T, et al. Effect of Wuziyanzong Pill medicated serum on secretion of rat sertoli cells in vitro. Chin J Tradit Chin Med Pharm 2013; 28:631-4.

19. Xu YP, Liu BX, Zhang XP, Yang CW, Wang CH. A Chinese herbal formula, Wuzi Yanzong pill, improves spermatogenesis by modulating the secretory function of Sertoli cells. Chin J Integr Med 2014;20:194-9.

20. Ke M, Liu B, Wang C, Pei X. Study on the effects of Wuzi Yanzong pill on semen quality and its related mechanism. Chin J Androl 2016;30:30-3.

21. Hu S, Li Y, Cao Y, Jian G, Guo J. Influence and mechanism of Wuzi Yanzong Wan on proliferation of Sertoli cells in infant rats. J Beijing Univ Tradit Chin Med 2017;40:917-22.

22. Wang QZ, Wang DF, Liu HL, Feng DJ, Wang HH, Guo ZB. Effects of extracts from Liuwei dihuang Pill on the proliferation of mouse Sertoli cells. Lishizhen Med Mat Medica Res 2013;24:1363-5.

23. Gu Y, Zhang X, Sun D, Zhao H, Cai B, Gao C, et al. The stimulative effect of Yangjing capsule on testosterone synthesis through Nur77 pathway in Leydig cells. Evid Based Comple- ment Altern Med 2015;2015:1-8.

24. Xu Y, Wu B, Jiang Y. Effect of icariin on the proliferation, apoptosis and testosterone synthesis of immature rat Leydig cells. Mod J Integr Tradit Chin West Med 2013;22:2864-6.

25. Nan Y, Zhang X, Yang G, Xie J, Lu Z, Wang W, et al. Icariin stimulates the proliferation of rat Sertoli cells in an ERK1/2dependent manner in vitro. Andrologia 2014;46:9-16.

26. Chen M, Hao J, Yang Q, Li G. Effects of icariin on reproductive functions in male rats. Molecules 2014;19:9502-14.

27. Wu ZQ, Chen DL, Lin FH, Lin L, Shuai O, Wang JY, et al. Effect of bajijiasu isolated from Morinda officinalis F. C. how on sexual function in male mice and its antioxidant protection of human sperm. J Ethnopharmacol 2015;164:283-92.

28. Yang X, Ding CF, Zhang YH, Yan ZZ, Du J. Extract from Cuscuta chinensis against the structure of human sperm membrane and the oxidative injury of function. Chin Pharm J 2006;41:515-8.

29. Shi GJ, Zheng J, Wu J, Qiao HQ, Chang Q, Niu Y, et al. Beneficial effects of Lycium barbarum polysaccharide on spermatogenesis by improving antioxidant activity and inhibiting apoptosis in streptozotocin-induced diabetic male mice. Food Funct 2017;8:1215-26.

30. Yu HY, Chen ZY, Sun B, Liu J, Meng FY, Liu Y, et al. Lignans from the fruit of Schisandra glaucescens with antioxidant and neuroprotective properties. J Natl Prod 2014;77:1311-20.

31. Yin JL, Xu Y, Wu B. Wuziyanzong compound relieves oxidative stress injury and inhibits the apoptosis of Sertoli cells. J Natl Androl 2013;19:257-61.

32. Li WL, Dai Y, Xu D, Ji YB. Effects of different polar fractions from Jinkuishenqiwan on testosterone and oxidative stress in rats with kidney-yang deficiency induced by hydrocortisone. Chin J New Drugs 2007;16:1944-6.

33. Zhou SH, Wen ZW, Liang AJ, Zhang ST. Experimental research on therapeutic efficacy of traditional chinese medicine Shengjing Capsule extracts in treating spermatogenesis impairment induced by oxidative stress. Med Sci Monit 2016;22: 50-6.

34. Weng Z, Zhou S, Liang A, Li L, Li TL, Zhang S. Effects of traditional Chinese medicine on spermatogenesis damage of rats caused by cigarette smoke exposure. Chin J Androl 2016;33: 11-7.

35. Meng X, Lindahl M, Hyvönen ME, Parvinen M, de Rooij DG, Hess MW, et al. Regulation of cell fate decision of undifferentiated spermatogonia by GDNF. Science 2000;287:1489-93.

36. Yang WM, Kim HY, Park SY, Kim HM, Chang MS, Park SK. Cynomorium songaricum induces spermatogenesis with glial cell-derived neurotrophic factor (GDNF) enhancement in rat testes. J Ethnopharmacol 2010;128:693-6. 
37. Jin B, Cai B, Sun D, Zhang X, Cui Y, Deng W, et al. Yangjing capsule extract promotes proliferation of GC-1 spg cells via up-regulated POU3F1 pathway. Biosci Trends 2017;11:95104.

38. Xu W, Luo Z, Jin J, Wang Q, Jiang Y, Han B, et al. Establishment of cyclophosphamide-induced oligopermia mouse model and the curative effect of matrimony vine. Chin J Androl 2015;29:3-7.

39. Zhu Q, Xiao L, Yang J, Hou L, Zhang D. Effects of Jinguishenqi bolus on apoptosis of kidney-yang deficiency model of testicular cells from the Fas/FasL pathway. Lishizhen Med Materia Medica Res 2017;28:272-6.

40. Huang WF, Zhang CC, Liu J, Song LX, Peng B, Zhao HX. Protective effect of Wuzi Yanzong prescription on apoptosis in germ cells of mice induced by cyclophosphamide. Zhong Yao Cai 2016;39:1143-7.

41. Ma N, Zhao HX, Chen Q, Han GF, Liu ZC, Yuan D, et al. Protective effect of Wuzi Yanzong recipe on testicular DNA damage and apoptosis in natural ageing rats. Zhongguo Zhong Yao Za Zhi 2018;43:1675-81.

42. Zhang C, Wang Y, Xu K, Wang Z, Hou Y. Effect of different nourishing formulas on spermatogenic cell apoptosis of aging mice. Chin J Exp Tradit Med Formulae 2018;4:148-53.

43. Bray TM, Bettger WJ. The physiological role of zinc as an antioxidant. Free Radic Biol Med 1990;8:281-91.

44. Sharif R, Thomas P, Zalewski P, Fenech M. The role of zinc in genomic stability. Mutat Res 2012;733:111-21.

45. Flohé L. Selenium in mammalian spermiogenesis. Biol Chem 2007;388:987-95.

46. Lapointe S, Ahmad I, Buhr MM, Sirard MA. Modulation of postthaw motility, survival, calcium uptake, and fertility of bovine sperm by magnesium and manganese. J Dairy Sci 1996;79:2163-9.

47. Zhao J, Dong X, Hu X, Long Z, Wang L, Liu Q, et al. Zinc levels in seminal plasma and their correlation with male infertility: a systematic review and meta-analysis. Sci Rep 2016;6: 22386.

48. Scott R, MacPherson A, Yates RW, Hussain B, Dixon J. The effect of oral selenium supplementation on human sperm motility. Br J Urol 1998;82:76-80.

49. Yu NC, Guan JH. Study on trace elements in 8 kidney-tonifying Chinese medicines and their clinical efficacy. ShiZhen J Tradit Chin Med Res 1997;8:33-4.

50. Lin S, Zhao L, Dong S, An D. Determination of selenium in ten kinds of traditional Chinese medicine by fluorescence spectrophotometry. J China Pharm Univ 1989;20:46-47.

51. Zhou SH, Xie JX. The application of treating male diseases from the perspective of blood stasis. J New Chin Med 2007;
39:97-8

52. Dong W, Jin B, Sun D, Cai B, Deng W, Cui Y, et al. Bushenhuoxue prescription facilitates testicular microcirculation in dyszoospermia mice via activating VEGF/VEGFR2 pathway. Chin J Androl 2018;32:30-5.

53. Guo J, Wang F, Zhang Q, Geng Q, Yu GJ, Zhao JY, et al. Treatment of oligospermia/asthenozoospermia patients by three different Chinese medical principles: a randomized control clinical study. Chin J Integr Tradit West Med 2013;33:1170-3.

54. Chen D, Zhong J, Chen S, Zhang Y, Zhang W, Wang G, et al. Effect of supplemented Wuzi Yanzong decoction on the quality of sperm and secretion of seminal fructose. J Tradit Chin Med 2013;54:401-4.

55. Sun ZG, Lian F, Jiang KP, Zhang JW, Ma FM, Zhang N, et al. Shengjing prescription improves semen parameters of oligoasthenozoospermia patients: efficacy and mechanism. Natl J Androl 2012;18:764-7.

56. Shang XJ, Guo J, Chen L, Deng CH, Sun XZ, Geng Q, et al. Qilin pills for oligoasthenospermia: a multi-centered clinical trial. Natl J Androl 2011;17:1139-42.

57. Au C, Yeung W, Xu F. Meta-analysis on TCM diagnosis and treatment of oligoasthenospermia. Chin Arch Tradit Chin Med 2015;33:2268-73.

58. Zhou JF, Li Q, Zhang QH, Lin RW, Chen ZQ, Xiang ST. Kidney-tonifying Chinese medicine for male infertility: a systematic review of randomized controlled trials. Natl J Androl 2015;21:833-40.

59. Jerng UM, Jo JY, Lee S, Lee JM, Kwon O. The effectiveness and safety of acupuncture for poor semen quality in infertile males: a systematic review and meta-analysis. Asian J Androl 2014;16:884-91.

60. Purvis K, Christiansen E. Infection in the male reproductive tract. impact, diagnosis and treatment in relation to male infertility. Int J Androl 1993;16:1-13.

61. Lu F, He Q, Zhang B, Liu Z, Li L. Effects of Zhibai Dihuang decoction on expression of IL-2, TNF- $\alpha$ in the testis of rats infected by ureaplasma urealyticum. China J Tradit Chin Med Pharm 2011;26:448-50.

62. Upadhyaya M, Hibbard BM, Walker SM. Antisperm antibodies and male infertility. Br J Urol 1984;56:531-6.

63. Mahony MC, Alexander NJ. Sites of antisperm antibody action. Hum Reprod 1991;6:1426-30.

64. Li X, Yu X, Liu J, Liao X, Miao Y. An Experimental Study of the effect of Zhibai Dihuang Decoction on immunological infertility. Tradit Chin Drug Res Clin Pharmacol 1997;8:83-5.

65. Wang W, Huang Z, Tang M, Li X, Chen Y, Chan M, et al. A histological and immunohistochemical study on MianBu I \& II treating immunological infertility of male mice. Chin J 
Histochem Cytochem 2001;10:81-5.

66. Lun X. Effects of acutherapy on T lymphocyte subgroup classification in male immuno-infertility. J Beijing Univ TCM 2004;27:90-2.

67. Rajender S, Avery K, Agarwal A. Epigenetics, spermatogenesis and male infertility. Mutat Res 2011;727:62-71.

68. Boissonnas CC, Abdalaoui HE, Haelewyn V, Fauque P, Du- pont JM, Gut I, et al. Specific epigenetic alterations of IGF2H19 locus in spermatozoa from infertile men. Eur J Hum Genet 2010;18:73-80.

69. Lian F, Sun J, Guo L, Sun Z, Wu H. Effects of Shengjing Formula on the expression of H19 imprinted gene in infertile patients caused by oligoasthenospermia with kidney deficiency syndrome. J Tradit Chin Med 2014;55:1113-6. 